\title{
Magnetic Dipole Sum Rules for Odd-Mass Nuclei
}

\author{
J.N. Ginocchio ${ }^{1,3}$ and A. Leviatan ${ }^{2,1,3}$ \\ 1 Theoretical Division, Los Alamos National Laboratory, Los Alamos, New Mexico 87545, USA \\ ${ }^{2}$ Racah Institute of Physics, The Hebrew University, Jerusalem 91904, Israel \\ ${ }^{3}$ European Centre for Theoretical Studies in Nuclear Physics and Related Areas (ECT*), I-38050 \\ Villazano, Trento, Italy
}

\begin{abstract}
Sum rules for the total- and scissors-mode M1 strength in odd-A nuclei are derived within the single-j interacting boson-fermion model. We discuss the physical content and geometric interpretation of these sum rules and apply them to ${ }^{167} \mathrm{Er}$ and ${ }^{161} \mathrm{Dy}$. We find consistency with the former measurements but not with the latter.
\end{abstract}

21.60.Fw, 21.10.Re, 23.20.-g, 27.70.+q

Typeset using REVTEX 
The orbital magnetic dipole scissors mode [1] has by now been established experimentally as a general phenomenon in deformed even-even nuclei [2]. The M1 strength systematics and deformation dependence have been extensively measured and corroborated in a variety of sum rules [3, 4. Within the interacting boson model (IBM) [5], a sum rule [3] has related this strength to the number of quadrupole bosons in the ground state of the even-even target nucleus, $\left\langle 0\left|N_{d}\right| 0\right\rangle$,

$$
\sum_{f} B\left(M 1: 0^{+} \rightarrow 1^{+}, f\right)=\frac{3}{4 \pi} g_{v}^{2} \frac{6 N_{\pi} N_{\nu}}{N(N-1)}\left\langle 0\left|N_{d}\right| 0\right\rangle .
$$

Here $g_{v}=\left(g_{\pi}-g_{\nu}\right), g_{\rho}$ are the proton $(\rho=\pi)$ and neutron $(\rho=\nu)$ boson g-factors and $N_{\rho}$ the corresponding boson numbers, $N=N_{\pi}+N_{\nu}$. For deformed nuclei $\left\langle 0\left|N_{d}\right| 0\right\rangle$ can be expressed in terms of the deformation determined from $\mathrm{B}(\mathrm{E} 2)$ values, and the measured M1 strength was shown to be in good agreement with this sum rule [6]. A survey [7] of scissors states in deformed odd-mass nuclei within the framework of the interacting boson-fermion model (IBFM) [8] predicted strong fragmentation and sizeable symmetric (single-particle dominated) and non-symmetric (scissors) M1 strength. Initial measurements on odd nuclei (Dy, Gd, Tb) indicated missing $\mathrm{B}(\mathrm{M} 1) \uparrow$ strength compared to the strength observed in the neighboring even-even nuclei 9 [1]. On the other hand, in ${ }^{167} \mathrm{Er}$ these M1 strengths were found to be comparable, with appreciable contribution coming from higher energies not accessed in previous experiments [12]. Inspired by these puzzling results we have derived sum rules for the total- and scissors mode M1 strength in odd nuclei within the IBFM. We focus on odd nuclei with the fermion in an orbital with single-nucleon angular momentum $\mathrm{j}$, which, for practical purposes, means that our sum rules are applicable to nuclei for which the ground state has the fermion filling the intruder (abnormal) orbit. In general, we find that the sum rules measure two quantities, the average value of $N_{d}$ in the ground state and the average relative orientation of the single-particle and total angular momenta. We apply the formalism to ${ }^{161} \mathrm{Dy}$ and ${ }^{167} \mathrm{Er}$ having an odd neutron in the $1 i_{13 / 2}$ orbital.

We begin by defining the weak coupling basis in which a nucleon with angular momentum $j$ is coupled to a bosonic wave-function $\left|\left[\alpha, F, F_{0}, L\right], j ; J, M\right\rangle$ specified by the $F$-spin [13] $F$, 
its projection, $F_{0}=\left(N_{\pi}-N_{\nu}\right) / 2$, the angular momentum of the IBM core $L$, and additional labels $\alpha . J$ is the total angular momentum (projection $M$ ) resulting from the coupling of $L$ and $j$. The initial ground state wave function will then be a linear combination of these states,

$$
|i ; J\rangle=\sum_{L} C_{i, L}\left|\left[\alpha_{i}, F_{m}, F_{0}, L\right], j ; J M\right\rangle
$$

with $F_{m}=N / 2$ the maximal $F$-spin and the label $i$ indicates all quantum numbers that may be needed to specify uniquely the initial state. Throughout this discussion we assume that the boson $F$-spin is conserved. The magnetic dipole operator is given by,

$$
T_{m}=g_{\pi} \hat{L}_{\pi, m}+g_{\nu} \hat{L}_{\nu, m}+g_{j} \hat{j}_{m}=g \hat{J}_{m}+g_{v} \hat{L}_{v, m}+g_{F} \hat{j}_{m}
$$

where $\hat{J}_{m}=\hat{L}_{m}+\hat{j}_{m}, \hat{L}_{m}=\hat{L}_{\pi, m}+\hat{L}_{\nu, m}$ and $\hat{j}_{m}$ are the total-, IBM core-, and singlenucleon angular momentum operators respectively, $\hat{L}_{v, m}=\left(\hat{L}_{\pi, m}-\hat{L}_{\nu, m}\right) / 2$ with $\hat{L}_{\rho, m}$ the individual boson angular momenta operators, $g_{j}$ is the nucleon g-factor, and $g=\left(g_{\pi}+g_{\nu}\right) / 2$, $g_{v}=g_{\pi}-g_{\nu}, g_{F}=g_{j}-g$. The operators $\hat{J}_{m}, \hat{L}_{m}$ and $\hat{j}_{m}$ are F-spin scalars $\left(F=F_{0}=0\right)$ and contribute only to symmetric $\rightarrow$ symmetric transitions $\left(F_{m} \rightarrow F_{m}\right)$. The $\hat{L}_{v}$ operator is F-spin vector $\left(F=1, F_{0}=0\right)$ and contribute also to symmetric $\rightarrow$ non-symmetric (scissors) transitions $\left(F_{m} \rightarrow F_{m}-1\right)$ [7]. The total B(M1) strength from the ground state to all final states is given by,

$$
\sum_{f} B(M 1: i \rightarrow f)=\frac{3}{4 \pi} \frac{\sum_{f}\left|\left\langle i ; J|| T|| f ; J_{f}\right\rangle\right|^{2}}{(2 J+1)}=\frac{3}{4 \pi}\langle i ; J|T \cdot T| i ; J\rangle
$$

where the dot in $T \cdot T$ denotes a scalar product and $\left\langle i ; J\|T\| f ; J_{f}\right\rangle$ is a reduced matrix element (r.m.e.). By using tensor operator identities [14] and the Wigner-Eckart theorem in F-spin space, we can evaluate the matrix elements of $T \cdot T$ in the weak coupling basis. Specifically, $T \cdot T$ is a sum of terms of the form $\hat{L} \cdot \hat{L}, \hat{j} \cdot \hat{j}, \hat{L} \cdot \hat{j}, \hat{L} \cdot \hat{L}_{v}, \hat{L}_{v} \cdot \hat{j}$ and $\hat{L}_{v} \cdot \hat{L}_{v}$. States in the weak coupling basis are eigenstates of the first three terms. The matrix elements of $\hat{L}_{v} \cdot \hat{j}$ reduce to a r.m.e. of the angular momentum $\hat{j}$ in the fermion space (which is known) times the r.m.e. of $\hat{L}_{v}$ between boson states with $\left(F_{m}, F_{0}\right)$. The bosonic matrix element is related 
by the Wigner Eckart theorem to a matrix element between states with $\left(F_{m}, F_{0}=F_{m}\right)$ for which $\hat{L}_{v}$ become the total boson angular momentum in the boson space, whose matrix elements are diagonal and known. Similar F-spin reduction appear in the matrix elements of the F-spin vector $\hat{L} \cdot \hat{L}_{v}$ term. Likewise, we can evaluate the $\hat{L}_{v} \cdot \hat{L}_{v}$ contribution by decomposing it to $F$-spin components $\left(F=2, F_{0}=0\right)$ and $\left(F=F_{0}=0\right)$, in the same way that was done for even-even nuclei in [3]. The relevant $F$-spin Clebsch Gordan Coefficients (C.G.C.) induce particular $N_{\rho}$-dependence for each term. The resulting matrix elements of $T \cdot T$ in the weak-coupling basis are diagonal in $\mathrm{L}$ and by averaging them over $\sum_{L} C_{i, L}^{2}$ we can evaluate the right hand side of Eq. (凷).

Consider first the $M 1$ strength from the ground to the scissors states. This involves symmetric $\rightarrow$ non-symmetric transitions with a change of $F$-spin: $F_{m} \rightarrow F_{m}-1$, and hence are induced only by the isovector operator $g_{v} \hat{L}_{v}$. Consequently, their contribution to the summed strength is proportional to the $F$-spin C.G.C. $g_{v}^{2}\left(F_{m}-1, F_{0} ; 1,0 \mid F_{m}, F_{0}\right)^{2}=2 g_{v}^{2} N_{\pi} N_{\nu} / N(N-$ 1). This dependence provides the signature needed to identify the contribution of these transitions to the right hand side of Eq. (四), and thus leads to the following sum-rule for the M1 strength from the ground state to the scissors mode $(s c)$ in odd-A nuclei,

$$
\sum_{f} B(M 1: i \rightarrow s c, f)=\sum_{f} B(M 1: i \rightarrow f)_{c o r e} .
$$

Here

$$
\sum_{f} B(M 1: i \rightarrow f)_{c o r e}=\frac{3}{4 \pi} g_{v}^{2} \frac{N_{\pi} N_{\nu}}{N(N-1)}\left[6\left\langle N_{d}\right\rangle-\frac{\langle L(L+1)\rangle}{N}\right],
$$

and

$$
\begin{aligned}
\left\langle N_{d}\right\rangle & =\sum_{L} C_{i, L}^{2}\left\langle\left[\alpha_{i}, F_{m}, F_{0}, L\right], j ; J M\left|N_{d}\right|\left[\alpha_{i}, F, F_{0}, L\right], j ; J M\right\rangle, \\
\langle L(L+1)\rangle & =\sum_{L} C_{i, L}^{2} L(L+1) .
\end{aligned}
$$

The first term in expression (6) is similar to that in Eq. (11), except that in the latter $\left\langle 0\left|N_{d}\right| 0\right\rangle$ is the average number of quadrupole bosons in the ground state of the even-even nucleus with boson angular momentum zero $(L=0)$, whereas $\left\langle N_{d}\right\rangle$ in Eqs. (6)-(7) is the 
average number of quadrupole bosons in the core of the neighboring odd nucleus which will have an admixture of boson angular momenta. However, for deformed nuclei in the large $\mathrm{N}$ limit, $\left\langle N_{d}\right\rangle / N$ is independent of the boson angular momentum to order $\mathcal{O}(1 / N)$ [15]. The $L$-dependent correction to $\left\langle N_{d}\right\rangle / N$ of $\mathcal{O}\left(1 / N^{2}\right)$ has the same dependence on the average angular momentum square, $\langle L(L+1)\rangle$, as the second term in (6), and reduces the magnitude of this $\mathcal{O}\left(1 / N^{2}\right)$ correction. Hence we suggest that the two terms in Eq. (6) constitute the contribution of the core to the total B(M1) and can be calculated in the way outlined in [6] to order $\mathcal{O}(1 / N)$ using the deformation of the odd nucleus. To this order, the sum rule in Eq. (5) states that the summed M1 strength from the ground to the scissors mode in an odd nucleus exhibits quadratic dependence on the Bohr-Mottelson quadrupole deformation [6] and is equal to the summed $B\left(M 1: 0^{+} \rightarrow 1^{+}\right)$strength in the neighbouring even-even nucleus. Adapting the criteria of [16] for assigning measured M1 strength to the scissors mode, we obtain the values for $B(M 1)_{\text {core }}$ which are $2.42(0.18) \mu_{N}^{2}$ from ${ }^{160}$ Dy [17] and 2.67(0.19) $\mu_{N}^{2}$ from ${ }^{166} \operatorname{Er}$ [18]. The measured scissors strength is $0.71(0.10) \mu_{N}^{2}$ in ${ }^{161} \mathrm{Dy}$ [10] and 3.14(1.12) $\mu_{N}^{2}$ in ${ }^{167} \operatorname{Er}$ [12] assuming M1 transitions. Referring to the sum rule of Eq. (5), these results indicate significant lack of M1 scissors strength in ${ }^{161}$ Dy and consistency, within the experimental errors, for ${ }^{167} \mathrm{Er}$.

To obtain a sum rule for the total M1 strength in odd nuclei we need to consider the contributions of all terms in Eq. (4). We note, however, that unlike even-even nuclei, there is a magnetic dipole transition to the ground state proportional to the magnetic moment. This elastic transition is not measured in $\left(e, e^{\prime}\right)$ and $\left(\gamma, \gamma^{\prime}\right)$ experiments, which employ continuous wave beams (Bremsstrahlung); hence we subtract it and obtain

$$
\sum_{f \neq i} B(M 1: i \rightarrow f)=\frac{3}{4 \pi}\left[\langle i ; J|T \cdot T| i ; J\rangle-\frac{|\langle i ; J|| T|| i ; J\rangle|^{2}}{(2 J+1)}\right]
$$

The subtraction in Eq. (8) results in partial cancellation of terms and we finally arrive at the following sum rule

$$
\sum_{f \neq i} B(M 1: i \rightarrow f)=\sum_{f} B(M 1: i \rightarrow f)_{\text {core }}+\frac{3}{4 \pi}\left(g_{j}-\bar{g}\right)^{2} j(j+1)\left[1-\langle\cos \theta\rangle^{2}\right]
$$


where $\bar{g}=\left(N_{\pi} g_{\pi}+N_{\nu} g_{\nu}\right) / N$ is the weighted boson g-factor and

$$
\langle\cos \theta\rangle=\frac{j(j+1)+J(J+1)-\langle L(L+1)\rangle}{2 \sqrt{j(j+1) J(J+1)}} .
$$

The right hand side of Eq. (10) is the semi-classical expression 14 for the cosine of the angle $\theta$ between the vectors $\vec{j}$ and $\vec{J}$ (see Fig. 1). The sum rule, therefore, provides information on the relative orientation of these angular momenta which, in turn, as seen from Eq. (10), depends on the average angular momenta square $\langle L(L+1)\rangle$ of the core. From (9), we see that the single-nucleon contribution to the B(M1) vanishes for both total alignment and anti- alignment $(\theta=0, \pi)$, and is maximum when $\vec{j}$ and $\vec{J}$ are perpendicular $(\theta=\pi / 2)$. The particular $j(j+1)\left[1-\langle\cos \theta\rangle^{2}\right]$ dependence is intuitively understood [19] from the geometry of the angular momenta shown in Fig. 1. For the magnetic transition strength only the component of $j$ perpendicular to $J\left(j_{\perp}=j \sin \theta\right)$ is effective (oscillating dipole as $\vec{j}$ precesses about $\vec{J})$, whereas the parallel component $\left(j_{\|}=j \cos \theta\right)$ contributes only to the static moment (see Eq. (11) below). The core contribution in Eq. (9) is determined by the same procedure discussed for the scissors mode sum rule of Eq. (5). The parameters $\bar{g}$ and $\langle\cos \theta\rangle$ can be determined from the magnetic moments of the odd nucleus $\left(\mu_{J}\right)$ and the neighbouring even-even nucleus $\left(\mu_{L}\right)$,

$$
\begin{aligned}
& \mu_{L}=\bar{g} L, \\
& \mu_{J}=\left[\bar{g}+\left(g_{j}-\bar{g}\right) \sqrt{\frac{j(j+1)}{J(J+1)}}\langle\cos \theta\rangle\right] J .
\end{aligned}
$$

We take $g_{j}$ to be the Schmidt value $g_{j, S c h m i d t}$ or the quenched Schmidt $0.7 g_{j, S c h m i d t}$ [7].

For weak coupling, the ground state has $L=0$ and $J=j$, so that $\langle\cos \theta\rangle=1$ and hence

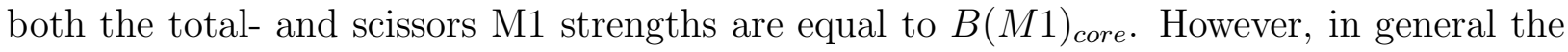
sum rule in Eq. (9) is an upper limit on the total B(M1) strength because the basis states of bosons coupled to a single-nucleon will be over-complete since the bosons represent coherent pairs of fermions some of which are occupying the single-nucleon orbital $\mathrm{j}$. Hence the sum rule which includes only Pauli allowed states (PA) will be in reality given by,

$$
\sum_{f \neq i} B(M 1: i \rightarrow f)_{P A}=\sum_{f \neq i} B(M 1: i \rightarrow f)-\sum_{f \neq i} B(M 1: i \rightarrow f)_{P F}
$$


To calculate the Pauli forbidden (PF) strength we need a model which we presently take to be the strong coupling limit of the IBFM [8,20]. In this limit, the amplitudes in Eq. (2) are proportional to a C.G.C. $C_{K, L, j, J}=\sqrt{2(2 L+1) /(2 J+1)}(L, 0 ; j, K \mid J, K)$ with $L$ even and $K$ is the projection of $j$ (and of $J$ ) along the symmetry axis (the corresponding projection of $L$ is zero). We can then calculate the required averages by using the fact that the expression for $\cos \theta$ in Eq. (10) can be related to a 6 -j symbol,

$$
\langle\cos \theta\rangle=(-1)^{j+J+L+1} \sqrt{(2 j+1)(2 J+1)}\left\langle\left\{\begin{array}{lll}
L & j & L \\
1 & J & j
\end{array}\right\}\right\rangle
$$

and then use well-known identity for summing a 6 -j times two C.G.C. [14. We find,

$$
\langle\cos \theta\rangle=\frac{2 K^{2}+(-1)^{j-J}(J+1 / 2)(j+1 / 2) \delta_{K, 1 / 2}}{\sqrt{J(J+1) j(j+1)}} .
$$

The transitions $K \rightarrow(K-1)$ are Pauli forbidden which gives $(K \neq 1 / 2)$,

$$
\sum_{f \neq i} B(M 1: i, K \rightarrow f, K-1)_{P F}=\frac{3}{8 \pi}\left(g_{j}-\bar{g}\right)^{2}[j(j+1)-K(K-1)] .
$$

We have evaluated the Pauli-corrected total M1 sum rule for the nuclei ${ }^{161}$ Dy $(j=13 / 2$, $J=5 / 2, K=5 / 2)$ and ${ }^{167} \operatorname{Er}(j=13 / 2, J=7 / 2, K=7 / 2)$, taking the experimental $B(M 1)_{c o r e}$ values as before from the neighboring even-even nuclei and using the total strength identified (assuming M1 transitions) [10,12]. From the magnetic moments of the first $2^{+}$states in ${ }^{160} \mathrm{Dy}$ and ${ }^{166} \mathrm{Er}$ we determine $\bar{g}=0.362 \mu_{N}$ and $0.318 \mu_{N}$, respectively. We then determined $\langle\cos \theta\rangle$ from the magnetic moments of ${ }^{161} \mathrm{Dy}\left(\mu_{J}=-0.480 \mu_{N}\right)$ and ${ }^{167} \operatorname{Er}\left(\mu_{J}=-0.56385 \mu_{N}\right)$ using the bare Schmidt value of $g_{j, S c h m i d t}=-0.2943 \mu_{N}$ for $1 i_{13 / 2}$ neutron orbital, and the quenched value, $0.7 g_{j, S c h m i d t}=-0.2060 \mu_{N}$. The results are summarized in Table I. We see that, whereas the ${ }^{167} \mathrm{Er}$ sum rule is consistent, within the experimental error, with the measured value of the total M1 strength, for ${ }^{161}$ Dy there is a large amount of missing strength, suggesting that ${ }^{161}$ Dy has "unexpected properties", not ${ }^{167} \mathrm{Er}$ as implied by the title of [12]. The missing scissors M1 strength in ${ }^{161}$ Dy may reside at higher energies (as encountered in ${ }^{167} \mathrm{Er}$ [12]) while the missing total M1 strength (singleparticle dominated) may also be at lower energies where the increased background limits 
the experimental sensitivity [11]. There are initial indications that a fluctuation analysis of the spectra can be used to fix the unresolved strength in the background and leads to comparable strengths [21].

The sum rules reported in this work rely on good $F$-spin symmetry. The validity of this assumption and the implications of breaking this symmetry on M1 transitions in odd nuclei was elaborated in [7], and shown that in the rare-earth region the effect is small. The utility of the large- $\mathrm{N}$ approximation was throughly investigated by the $1 / N$ technique (see 15 and references therein). Although we have confined the discussion to a single-j model space for the odd fermion (hence specific odd-mass nuclei), similar formulas should hold for a situation of pseudospin symmetry. Multi-j formulation of similar M1 sum rules requires confronting more allowed terms (and parameters) in the M1 operator and several $j$-amplitudes in the fermion wave-function.

In summary, we have derived sum rules for magnetic dipole transitions in odd nuclei with a nucleon in a single spherical orbit, $j$, assuming F-spin symmetry and large-N approximation for the boson core. The summed M1 strength from the ground to the scissors mode in such nuclei depends on the average number of quadrupole bosons in the ground state. This quantity, for large $N$, exhibits quadratic dependence on the Bohr-Mottelson quadrupole deformation and can be determined from the scissors M1 strength in the neighboring eveneven nucleus or from the deformation of the odd nucleus to $\mathcal{O}(1 / N)$. The total M1 strength depends in addition also on the average of the cosine of the angle between the odd nucleon angular momentum and the total angular momentum. This quantity is related to the average of the core angular momentum, $\langle L(L+1)\rangle$, and can be determined from the magnetic moments of the target and the neighboring even-even nucleus. In general this sum rule will be an upper limit from which the strength to Pauli forbidden states needs to be subtracted. In well-deformed nuclei the strong coupling limit can be used for that purpose. In comparing with the known $\mathrm{B}(\mathrm{M} 1)$ strength, we find consistency in ${ }^{167} \mathrm{Er}$ for the scissors- and for the total strength. However, we conclude that a substantial portion of the magnetic dipole scissors and total strengths are missing in ${ }^{161}$ Dy. Clearly, more measurements both at 
higher and lower energies are needed in ${ }^{161} \mathrm{Dy}$ to see if the predictions of the sum rules are satisfied. More and detailed calculations are needed to understand M1 properties in odd-mass nuclei. In particular, further theoretical attention is needed for including Pauli corrections in estimates of M1 strength and for understanding the different fragmentation patterns observed in different odd nuclei.

We are indebted to U. Kneissl, P. von Neumann-Cosel and F. Iachello for helpful discussions. We thank the European Centre for Theoretical Studies in Nuclear Physics and Related Areas (ECT*) for its kind hospitality during a stay from which this article originated. This work was supported in part by a grant from the Israel Science Foundation and by the U.S. Department of Energy. 


\section{REFERENCES}

[1] D. Bohle et al., Phys. Lett. B137, 27 (1984).

[2] For recent reviews see A. Richter, Prog. Part. Nucl. Phys. 34, 261 (1995);

U. Kneissl, H.H. Pitz and A. Zilges, Prog. Part. Nucl. Phys. 37, 349 (1996).

[3] J.N. Ginocchio, Phys. Lett. B265, 6 (1991).

[4] K. Heyde and C. De Coster, Phys. Rev. C44, R2262 (1991);

E. Moya de Guerra and L. Zamick, Phys. Rev. C47, 2604 (1993);

N. Lo Iudice and A. Richter, Phys. Lett. B304, 193 (1993).

[5] F. Iachello and A. Arima, The Interacting Boson Model (Cambridge University Press, Cambridge, 1987).

[6] P. von Neumann-Cosel, J.N. Ginocchio, H. Bauer and A. Richter, Phys. Rev. Lett 75, 4178 (1995).

[7] P. Van Isacker and A. Frank, Phys. Lett. B225, 1 (1989);

A. Frank, J. M. Arias and P. Van Isacker, Nucl. Phys. A531, 125 (1991).

[8] F. Iachello and P. Van Isacker, The Interacting Boson-Fermion Model (Cambridge University Press, Cambridge, 1991).

[9] I. Bauske, et al., Phys. Rev. Lett. 71, 975 (1993).

[10] J. Margraf, et al., Phys. Rev. C52, 2429 (1995).

[11] A. Nord et al., Phys. Rev. C54, 2287 (1996).

[12] C. Schlegel, P. von Neumann-Cosel, A. Richter and P. Van Isacker, Phys. Lett. B375, $21(1996)$.

[13] T. Otsuka, A. Arima, F. Iachello and I. Talmi, Phys. Lett. B76, 139 (1978).

[14] D. A. Varshalovich, A. N. Moskalev, V.K. Khersonskii, Quantum Theory of Angular 
Momentum (World Scientific, Singapore, 1988).

[15] S. Kuyucak and S. C. Li, Phys. Lett. B349, 253 (1995).

[16] N. Pietralla et al., Phys. Rev. C52, R2317 (1995).

[17] C. Wesselborg, et al., Phys. Lett. B207, 22 (1988).

[18] A. Maser et al., Phys. Rev. C53, 2749 (1996).

[19] F. Doüau, Nucl. Phys. A471, 469 (1987).

[20] A. Leviatan, Phys. Lett. B209, 415 (1988).

[21] J. Enders, N. Huxel, P. von Neumann-Cosel and A. Richter, to be published. 


\section{TABLES}

TABLE I. The total B(M1) strength in units of $\left(\mu_{N}^{2}\right)$ for ${ }^{161}$ Dy and ${ }^{167}$ Er for bare and quenched Schmidt $g_{j}$ values are tabulated. $\langle\cos \theta\rangle$ is defined in (10), $\sum B(M 1)$ is the total contribution of the sum rule (9), $\sum B(M 1)_{P F}$ is the contribution of the Pauli forbidden states in the strong coupling limit (15),$\sum B(M 1)_{P A}$ is the total strength of the Pauli allowed states (12), and $\sum B(M 1)_{\exp }$ are the measured values given in 10,12, respectively. Choice of parameters is discussed in the text.

\begin{tabular}{ccccccc}
\hline \hline Nucleus & $g_{j}$ & $\langle\cos \theta\rangle$ & $\sum B(M 1)$ & $\sum B(M 1)_{P F}$ & $\sum B(M 1)_{P A}$ & $\sum B(M 1)_{\exp }$ \\
${ }^{161} \mathrm{Dy}$ & $g_{j, \text { Schmidt }}$ & 0.358 & $6.78(0.18)$ & 2.31 & $4.47(0.18)$ & $0.88(0.13)$ \\
${ }^{161} \mathrm{Dy}$ & $0.7 g_{j, \text { Schmidt }}$ & 0.413 & $5.53(0.18)$ & 1.73 & $3.80(0.18)$ & $0.88(0.13)$ \\
${ }^{167} \mathrm{Er}$ & $g_{j, \text { Schmidt }}$ & 0.445 & $6.16(0.19)$ & 1.78 & $4.38(0.19)$ & $3.49(1.15)$ \\
${ }^{167} \mathrm{Er}$ & $0.7 g_{j, \text { Schmidt }}$ & 0.520 & $5.00(0.19)$ & 1.31 & $3.69(0.19)$ & $3.49(1.15)$ \\
\hline \hline
\end{tabular}




\section{FIGURES}

FIG. 1. Angular momenta for a particle coupled to an axially-symmetric core. $J, L$ and $j$, are the total-, core-, and single-particle angular momenta respectively. $K$ is the projection of $j$ (and of $J$ ) on the symmetry axis. 


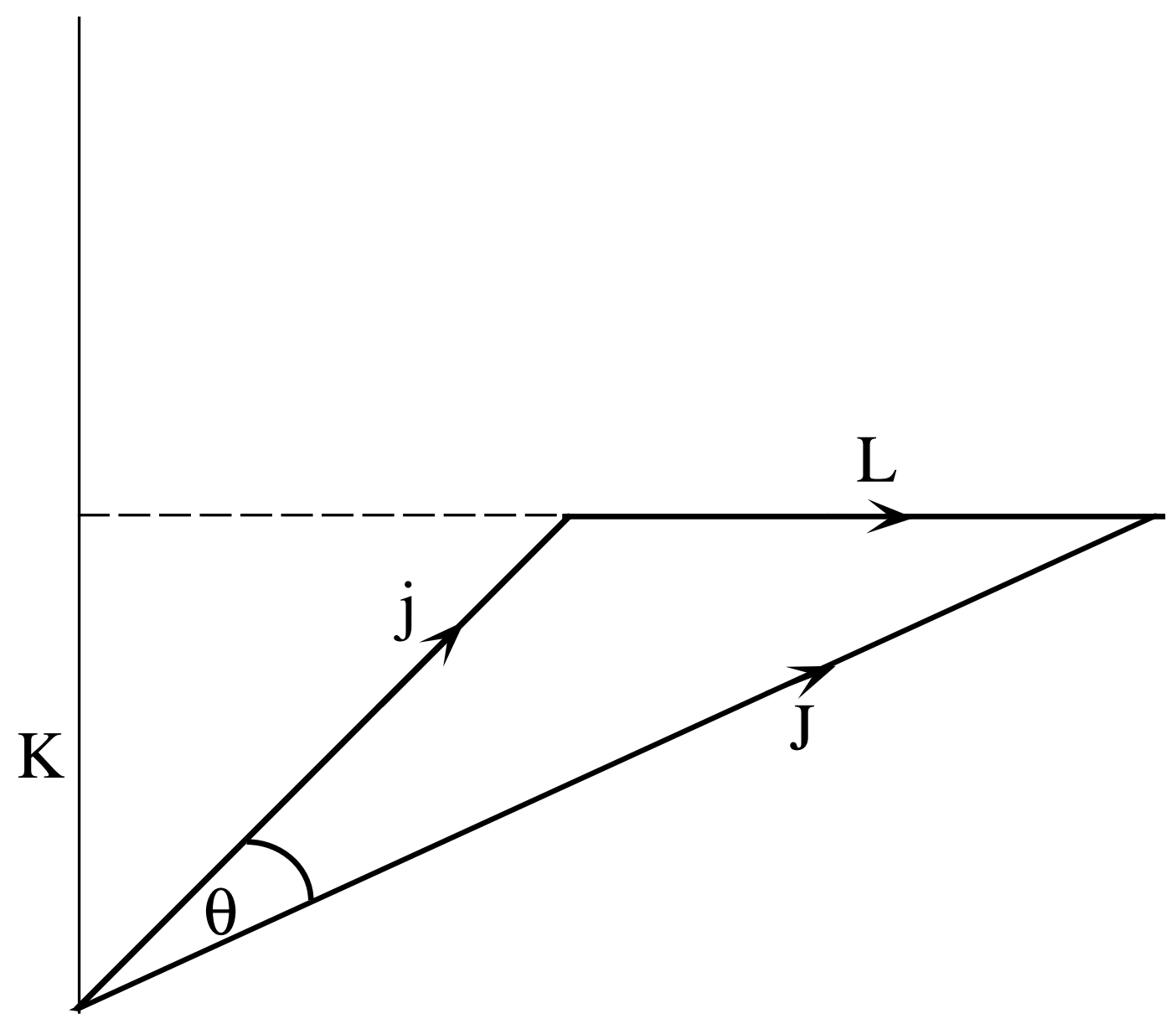

